\title{
Urge Incontinence and the Nervous System
}

\section{Koch WFRM ${ }^{1}$ and Marani E $^{2 *}$}

${ }^{1}$ Department of Urology, Noord-West Ziekenhuisgroep, The Netherlands

${ }^{2}$ Department of Biomedical Signals and Systems, University of Twente, The Netherlands

\section{Introduction}

"One should emphasize that urinary incontinence is a symptom, and not a definitive diagnosis, which means that unless the reason behind the symptom is diagnosed, correct therapy cannot be carried out [1]. In cases were the diagnosis remains unknown only the symptoms are treated. Commonly urinary incontinent patients present themselves for the first time in a primary health care setting. Urge incontinence is a very inconvenient symptom, urging the patient to see his or her primary care physician. These patients do expect that a correct diagnosis is made by the physician. Therefore some in depth knowledge of underlying neurology and pathology is needed.

Urge incontinence is defined by de International Continence Society as "The observation of involuntary leakage of urine from the urethra synchronous with the sensation of a sudden, compelling desire to void that is difficult to defer". If no infection or obvious pathology can be found the term Over Active Bladder (OAB) is also used. Its epidemiologic characteristics are a prevalence of $2.9 \%$ in France $6 \%$ in the Netherlands up to $19.9 \%$ in Turkey [2]. Most cited articles estimate a prevalence of between 12 and $17 \%$ [2]. A strong variability of the prevalence is noticed in different countries, probably due to the underlying risk factors and most likely also to social and/or economic factors. Urge incontinence in most studies shows an increasing prevalence with age. Risk factors are frequently badly studied due to: high drop-outs of patients in studies (by discomfort and/or shame of these patients); mostly small groups are studied, while often large groups are needed to get reliable results and it is practically impossible to perform double-blind randomized trials. Furthermore, available or tested drugs have side effects leading to a decreased patient compliance and therefore exclusion from studies. The risk factors that are acknowledged are: age, gender, obesity, life style, race, socio-economic status, reproductive factors and pelvic surgery. Attention is also given to specific conditions like: benign prostatic hyperplasia, pelvic organ prolapse, mental health, neurological diseases and diabetes [2].

This article presents a bird's-view of the neurological systems involved in micturition. We discuss some typical pathologic conditions often seen in primary health care, which have the common disabling symptom of urge incontinence. One should note that there are two possible origins of $\mathrm{OAB}$ symptoms: decreased capacity to handle the efferent signals in the brain and abnormally increased efferent signals from the bladder and/or the urethra [3].

\section{Urge Incontinence: Related Neuroanatomy and Physiology}

Onufrowicz (1863-1930) studied, in two human specimens, the sacral spinal cords. In between the medial and lateral motor areas in lamina IX of S1/S2 till S3/S4 he noticed a group of densely packed smaller neurons, which he called "nucleus X" [4]. "The group "X" which we found practically concerned to the second sacral segment, suggests by its circumscribed character some special function. From its location I should deem it possible and rather probable that it is a centre for some of the striated muscles which co-operate in the acts of erection and ejaculation, especially the ischiocavernosus or erector clitoridis and the bulbo-cavernosus or sphincter vaginae muscles" [5]. "He guessed incorrectly, however, that this cell group was not involved in innervating the urethral and anal sphincters" [6]. The cell group was then ignored for years in literature. Revived the importance of this nucleus for the preservation of bladder-rectal functions in amyotrophic lateral sclerosis (ALS) [7]. From then on the composition of comparable nuclei was studied in rat, cat, dog, rhesus monkey and man. First, only a somatic character was attributed to Onuf's nucleus related to the small striated pelvic muscles including the pelvic sphincters. The striated muscles of the external anal sphincter as well as from the smooth muscles of bladder and rectum were found Onuf-related and its dual character was stressed [8]. The fact that Onuf's nucleus was spared in Werdnig-Hoffmann Disease, healed poliomyelitis, Duchenne muscular dystrophy, spinal muscular atrophy and ALS while other (somatic) sacral motoneurons degenerated and that in Shy-Drager Syndrome (multiple system atrophy) and Fabry disease (both) Onuf's nucleus (and the parasympathetic nucleus) degenerated while somatic sacral motoneurons were spared, an autonomic character of the motoneurons of Onuf's nucleus was suggested [9].

A variety of passive electrical membrane properties of Onuf's motoneurons have been published: their low rheobase values and their lack of recurrent inhibition in spite of the presence of recurrent axon collaterals. The only significant difference between urethral sphincter motoneurons and anal sphincter motoneurons of Onuf's nucleus was the membrane time constant, which was twice as long in anal motoneurons compared to urethral motoneurons 9130 [10]. Moreover, casual apposition of their dendrites, the presence of crest-like synapses and electronic coupling (gap-junctions) are presumably responsible for fast signal transmission by facilitation in neighbouring motor neurons within the nucleus [11]. This means that with a minimum of activation a maximum of effect is achieved.

From this summing up it is at least clear that Onuf's nucleus is hard to classify as a somatic or an autonomic nucleus. Onuf's nucleus does not discern itself for its electric behaviour from somatic motoneurons, but it does by its ultrastructural configurations of crest-like contacts and gap-junctions. It is well established that Onuf's nucleus is connected to the anal and urethral external sphincters (Figure 1), the pelvic floor muscles and the ischiocavernosus and the bulbocavernosus muscles.

The autonomic parts steering the smooth muscles involved in micturition are well studied. The parasympathetic sacral intermediolateral nuclei S2-S4 and the T11-L2 sympathetic column are related to the sympathetic chain, and also to the plexus hypogastricus superior, the plexus pelvicus and the intramural ganglia. These structures govern the bladder and the smooth urethral musculature $[12,13]$. Supraspinal centres receive information from thoracolumbar and sacral

*Corresponding author: Marani E, Department of Biomedical Signals and Systems, University of Twente, The Netherlands, Tel: 0031614418505; E-mail: e.marani@utwente.nl

Received September 12, 2016; Accepted November 14, 2016; Published November 21, 2016

Citation: Koch WFRM, Marani E (2016) Urge Incontinence and the Nervous System. Primary Health Care 6: 246. doi:10.4172/2167-1079.1000246

Copyright: (C 2016 Koch WFRM, et al. This is an open-access article distributed under the terms of the Creative Commons Attribution License, which permits unrestricted use, distribution, and reproduction in any medium, provided the original author and source are credited. 


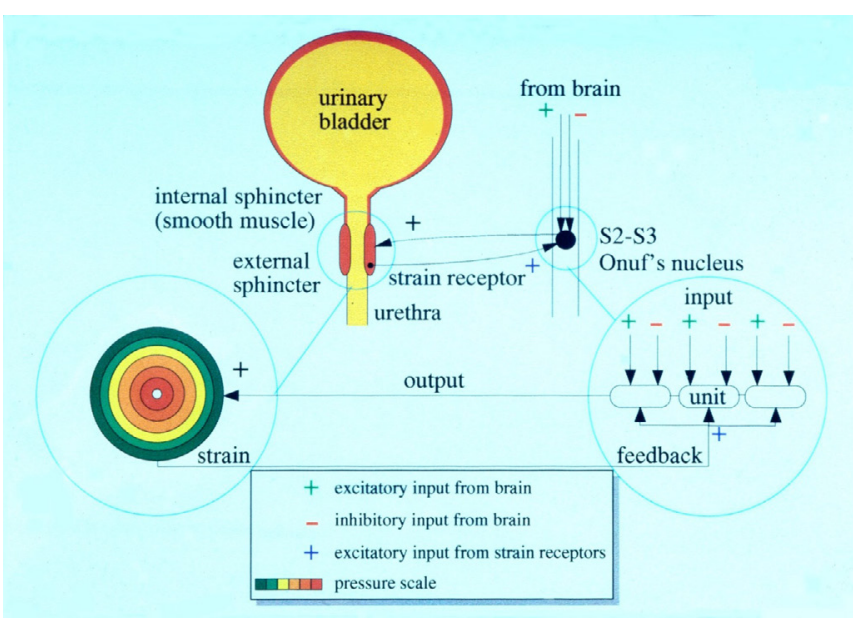

Figure 1: The role of Onuf's nucleus: Excitatory and inhibitory effects playing their role in the striated muscles of the urethra (courtesy Heldoorn et al. $[10,11,17])$.

sensory centres. $\mathrm{L}$ and $\mathrm{M}$ centres in the brainstem were proposed by Holtstege [14]. These pontine micturition centres should be subdivided by an $\mathrm{M}$ or parasympathetic related bulbar area (Barrington's nucleus) and an L or somatic bulbar area. Function for storage goes over the $\mathrm{L}$ area activating Onuf's nucleus and inhibiting the $\mathrm{M}$ region. The $\mathrm{M}$ region starts being active during voiding inhibiting the $\mathrm{L}$ region, thus relaxing the pelvic floor, and activating the sacral intermediolateral and paravesical ganglia to initiate a bladder contraction.

Sensory information of the urinary system reaches the preoptic area and thalamus, sometimes via mesencephalic centres (peri-aqueductal gray), from where this information is relayed to the cerebral cortex. Cortical information is relayed to both pontine levels and spinal cord levels. Physiologically this means that the cortical centres in general inhibit the pontine $M$ centre in order to avoid micturition. When micturition is needed cortical inhibition decreases and the $\mathrm{M}$ centre is activated and the L centre inhibited [15].

A short neuron system is working at the level of the bladder and the urethra sphincter network function [16,17]. Parasympathetic ganglia are located directly on the surface of the target organ, while sympathetic ganglia are localized paravertebral. Some exceptions are the pelvic plexus and the superior hypogastric ganglia, of which the last one is sympathetic but the pelvic plexus is considered mixed sympathetic and parasympathetic. In humans this system is involuntary. These peripheral ganglia are connected to each other establishing a local network that is capable to steer "simple" reflexes. This network belongs to the controller system that comprises a series of reflexes and regulates all pelvic reflexes, involving pelvic floor, sphincters, urogenital diaphragm and clitoris or penis $[3,18]$.

Need to void, start of voiding and micturition is organized by the urothelium. This is a multi-cell layer of epithelium that covers the inside of the uropoetic system, and smooth muscles of the bladder. They are responsible for the first activation of the nervous system. The urothelium will react on change in urine $\mathrm{pH}$, inflammation, mechanic stress and temperature changes [19]. The sensory neurotransmitters released from these epithelial cells can activate the sensory nerves in the bladder wall, the smooth muscle and/or the striated muscle. Beside the activation of nerves to inform ganglia and the spinal cord, there exists in the urethral wall an intrinsic system that seemingly is capable of reacting immediately to urethral threats. So, sphincter activity can be modulated by its urothelium. For storage of fluid that has to be expelled from the body, it is necessary to overcome any leakage from the bladder into the surrounding body compartments (the bladder has to be 'water tight'). The demands for performing such a task are high: permeability is extremely low; leakage between the epithelial cells is overcome by tight junctions between these epithelial cells. These tight junctions force any passage from urine constituents through the epithelial cells that actively can control such a passage to modulate the urine composition. This intricate function of the urine barrier must withstand extreme folding of the apical membrane during voiding and stretching during filling, which asks for specific lipids in the apical membranes of the epithelial cells $[19,20]$. So, the bladder is in fact a water-tight reservoir, despite any osmolaric differences between its surroundings. The urothelium also has a sensory function. The free nerve endings of pain afferent nerves contain several receptors, which can be activated by neurotransmitters that have been released by the urothelium (also nitric oxide that penetrates the nerve endings without receptor). The receptors involved are: purinergic-, vanilloid-, prostanoid-, and neurokinin receptors. Vanilloid receptors normally react at a temperature above $43^{\circ} \mathrm{C}$ and a $\mathrm{pH}$ lower than 6 . However, $\mathrm{pH}$ change can alter temperature sensibility of these receptors to body temperatures. The $\mathrm{pH}$ change occurs at inflammation. This alteration of $\mathrm{pH}$ is responsible for a shift in the threshold value of the sensory nerve endings causing a different voiding pattern [20]. This means clinically that in case of alteration in $\mathrm{pH}$ the voiding pattern changes: urinary tract infection for example causes change in urinary $\mathrm{pH}$ and therefore a change in voiding pattern (i.e., urgency and frequency).

In summary the urothelium is a specially designed layer of cells that protects the body against the urine it needs to expel. At the same time the urinary bladder controls the urine composition by its urothelial cells. Sensory input to the bladder is made possible by receptors measuring, e.g. urinary $\mathrm{pH}$, temperature, straining and torsion of the detrusor muscle cells. Thus, overshooting the fixed levels for each factor causes action of the bladder structures. For example if the maximum level of bladder wall strain is superseded the neurological pathway for bladder contraction, voiding and emptying is started.

\section{Micturition, Age and Neurodegenerative Diseases}

The prevalence of urinary incontinence increases with advancing age. Institution's residents are typically older and therefore prevalence in nursing homes is higher. In older women urge and mixed incontinence predominate [21]. The age related changes that are relevant for urge incontinence are present at different levels. At the level of bladder ultrastructure changes in the junction patterns between the urothelial cells are supposed to cause bladder over activity and thus urge incontinence. At the level of the bladder muscle axon degeneration can take place (especially of the sympathetic system fibres) that is responsible for impaired bladder contractility, increased residual urine and decreased functional bladder capacity. Due to the lack of oestrogens in most elderly female, mucosal thinning and lack of proteoglycans occur, reducing urethral wall opposition that extend up to the bladder trigone, causing irritation of sensory efferent nerves. This triggers detrusor over activity. Moreover there is a decrease of smooth and striated muscle fibres during aging in man and woman. Together with age related sensory neuropathy these alterations contribute to a higher prevalence of OAB. Postmenopausal lack of oestrogens has been associated with an increased incidence of urogenital atrophy and altered immune function of the epithelium being responsible for an increased likelihood of recurrent urinary tract infections and thus also causing urge incontinence [22]. 
Altered neurotransmitter concentrations and actions, especially in elderly, both central and peripheral, increase the likelihood of urinary tract dysfunction. Over 20 different types of neurotransmitters are discerned in the urinary system until now. Each year new ones are added to this list. Their function is often unknown and their receptors are still studied [3]. A few of the efferent receptors are well known: the cholinergic, purinergic, catecholaminergic, glutamatergic, glycinergic, GABA-ergic and nitric oxide receptors. Cholinergic and purinergic neurotransmitters produce the main excitatory effects. Since the amount of these neurotransmitters decreases with age a hypo-contractile bladder occurs responsible for an increase in postmicturition residue and therefore, a lower functional bladder capacity. This can lead to urgency, frequency and urinary incontinence. GABA release is responsible for inhibiting the detrusor over activity and thus plays a major role in inhibiting the micturition reflex. GABA is diminished during aging and, therefore, a decrease in the inhibiting function is found, causing $\mathrm{OAB}$ and urge incontinence [23].

The afferent system is important in the origin of OAB. The main function of the bladder is storage, which can be achieved by preventing contraction of the bladder smooth muscles. Switching to bladder contraction during micturition depends on sensory signals for pain (A $\delta$ and $C$ fibres), for stretch and for tension. Dysfunction of these signals leads to $\mathrm{OAB}$ and other symptoms like urgency, bladder pain and urge incontinence, because with aging the threshold of these receptors changes [24-26]. Remaining continence is under control of the fore brain, brainstem and spinal cord. With increasing age a higher prevalence of brain white matter hyper-intensities in the brain is observed, not only causing urge incontinence but also cognitive impairment and impaired bodily mobility, both contributing to disablement. Diseases that are related to pathology of the brain and brainstem are dementia and Parkinson's disease, whereas in early onset diseases like multiple sclerosis (MS) and multiple system atrophy (MSA) the spinal cord is also typically involved. In dementia supra-pontine lesions occur mostly by white matter degeneration, most striking in Alzheimer, and vascular impairment. All these supra-pontine diseases, including MS and MSA, are characterized by incontinence due to $\mathrm{OAB}$ or due to decreased inhibition of the micturition reflex [27].

Cerebro-vascular accidents (CVA) cause urinary tract dysfunction in up to $50 \%$ of patients with up to $30 \%$ having continued symptoms after 6 months of their CVA, of which OAB is the most common one. The likelihood of developing urinary tract dysfunction is highly associated with the location of the lesion. Brainstem strokes are accompanied by the highest incidence of OAB. Supra-pontine lesions are almost always associated with a bladder over activity, whereas diseases which also involve the spinal cord are characterized by a mixed over and under activity of the bladder [27-29]. A well-known disease of the central nervous system is Multiple Sclerosis (MS). MS causes inflammatory demyelinisation of nerve fibres, preventing normal neuro-signalling. Up to $90 \%$ of patients suffering from MS have urinary tract dysfunction: most likely $\mathrm{OAB}(65 \%)$, detrusor sphincter dissynergia $(35 \%)$ and detrusor under activity (25\%). The clinical presentation is often associated with a varying expression of urinary tract symptoms over the course of the disease. For example a patient can suffer from $\mathrm{OAB}$ and urge incontinence at one time, and later in time from detrusor under activity causing urinary retention $[28,29]$.

\section{Therapeutic Options}

Since urge incontinence is a symptom of underlying diseases, mostly of untreatable illnesses, the therapeutic options are seriously restricted. Treatment of the patient is limited to relieving the symptoms.
Medicinal treatment is targeted at the level of the receptors in the detrusor muscle. Normal bladder contraction in humans is mainly achieved by acetylcholine stimulation of muscarinic receptors [30]. The neurotransmitter acetylcholine acts on two classes of receptors, nicotinic and muscarinic ones. The muscarinic receptors are found within the detrusor smooth muscle, while nicotinic receptors are present in striated muscles. The subtype M3 of the muscarinic receptor is mainly involved in detrusor action. As a consequence the pharmaceuticals available are focussed on blocking the acetylcholine action on the (M3) muscarinic receptors [31]. This results in a decreased ability of the bladder contractility in OAB. Solifenacin, Darifenacin, Tolterodine and Mirabegron are typical examples of drugs with a specific antimuscarinic effect. The older drugs oxybutynine and flavoxate have a mixed action on the acetylcholine receptors. Oxybutynine has an anti-muscarinic effect, but also a muscle relaxant effect, and a local anaesthetic effect. Administration of the above mentioned drugs are unfortunately associated with anticholinergic side effects: obstipation, dry mouth and blurred vision. Research focusses on finding specific anti-M3 muscarinic drugs with minimum side effects and maximum effect and tolerability [32].

Botulinum toxin is a neurotoxin produced by Clostridium botulinum that consists of a heavy and light chain linked by a disulphide bond. In the synaptic cleft of the neuron the toxin binds to synaptic vesicle proteins by its heavy chain and is then internalized by the recycling of the synaptic membrane. The internalized toxin prevents acetylcholine release. Administration of this toxin to the detrusor muscle prevents exciting the muscarine receptors and thus bladder contractility is decreased. In the therapeutic setting of $\mathrm{OAB}$ the toxin is directly injected endoscopically in the detrusor muscle or submucosa. Typically the brands Botox and Dysport are used. Since the administration is performed in the target organ systemic side effects are extremely rare. It normally remains active for a period of 6-9 months; fortunately the administration can be easily repeated [33].

At the level of the peripheral nervous system two available treatments are discussed: PTNS (percutaneous posterior tibial nerve stimulation) formerly known as SANS (Stoller's Afferent Nerve Stimulation) and Neuromodulation by sacral nerve stimulation. In PTNS the optimal point to stimulate coincides with the Sp (spleen) 6 locus (Sanyinjiao) of the acupuncturist. In acupuncture, this site is considered to be significant for treatment of disturbances of the urogenital tract. In PTNS, a needle electrode is connected to an electrical stimulator generating 1-10 $\mathrm{mA}$ and placed against the tibial nerve in the ankle. $50 \%$ of the treated patients experience a reduction in voiding and urge episodes, in daytime and night time. The tibial nerve originates from the spinal nerves L4, L5, S1, S2 and S3. Hence, stimulation of the tibialis antidromically sends impulses to all these spinal segments, including the S2 and S3 branches (S3 mainly in males and S2, S3 in females) which constitute the nerves to the pelvic plexus and thus to the bladder. In this way, involuntary contractions of the bladder are also reduced. The mechanism by which this stimulation of the tibial nerve works is unknown. Factors that may be involved are the inhibitory interneurons at several spinal cord levels, and the descending pain-suppressing system. Termination of treatment results in return of incontinence [3]. In Sacral Nerve Stimulation, neuromodulation is carried out by stimulation of the third sacral segmental nerve (S3) in patients with urge incontinence and with therapy-resistant $\mathrm{OAB}$. Neuromodulation by sacral nerve stimulation requires placement of an electrode in the $\mathrm{S} 3$ foramen of the sacral bone. A dorsal approach makes it easy to identify the S3 foramen. The electrode is brought up to the ventral side of the opening against the $\mathrm{S} 3$ ventral ramus (for involved 
ventral rami of the sacral plexus [34]. This procedure is minimally invasive, as compared to Brindley's neurostimulation. The working mechanism of neuromodulation is unknown. For example a surprising effect of neuromodulation is decreasing bladder contractility in OAB, while the exact same treatment can cause an increased contractility in a hypo-contractile bladder. A suggested mechanism is somatic afferent inhibition of the sensory processing in the spinal cord [3].

\section{Conclusion}

Pelvic research is in arrear compared to other sciences. Restricted research of the morphology and of the mutual topography of the pelvic structures in combination with premature clinical application of the results explains the present state. The same mistake was repeatedly made, both in diagnosis and in the surgical treatment of urinary and faecal incontinence. Intricate connective tissue architecture, strongly related to incontinence, is present, which is largely neglected in anatomical research and in pelvic surgery. Urinary flow and pressure are determined by a series of partially unknown parameters and thus difficult to interpret. Smooth muscle electrical activity is related to relaxation and contraction both [13], which has been largely neglected. A better understanding of sphincter function and of the myo-cybernetic requirements of the lower urinary tract by mathematical models is needed $[10,11,35,36]$. Therefore, main focus should be on fundamental research, in order to understand the reasons behind the symptom of incontinence, followed by an effective translation of the results into clinical applications $[37,38]$.

\section{References}

1. Petros PE, Ulmsten U (1990) An integral theory of female urinary incontinence. Acta Obstet Gynecol Scandinavica 153: 1-79.

2. Abrams P, Cardozo L, Khoury S, Wine A (2013) Incontinence: International consultation on incontinence, $\left(5^{\text {th }}\right.$ ed).

3. Marani E, Koch WFRM (2014) The pelvis, structure, gender and society. Springer-Verlag, Berlin, Heidelberg.

4. Onufrowicz B (1899)Notes on the arrangement and function of the cell groups in the sacral region of the spinal cord. J Nerv Ment Dis 26: 498-504.

5. Onufrowicz B (1900) On the arrangement and function of the cell groups of the sacral region of the spinal cord in man. Arch Neurol Psychopathol 3: 387-411.

6. Lanska DJ (2002) Classic articles of $19^{\text {th }}$-century American neurologists: A critical review. J History Neurosci 11: 156-173.

7. Mannen T, Iwata M, Toyokura Y, Nagashima K (1977) Preservation of a certain motoneuron group of the sacral cord in amyotrophic lateral sclerosis: Its clinical significance. J Neurol Neurosurg Psychiatry 40: 464-469.

8. Yamamoto T, Satomi H, Ise H, Takatama H, Takahashi K (1978) Sacral spinal innervation of the rectal and vesical smooth muscles and the sphincteric striated muscles as demonstrated by the horseradish peroxidase method. Neurosci Lett 7: 41-47.

9. Marani E (2015) Nucleus of Onuf.

10. Heldoorn M, Marani E, Van Leeuwen J, Vanderschoot J (2003) A compartmental model of an external urethral sphincter motor neuron of Onuf's nucleus. Arch Phys Biochem 111: 193-201.

11. Heldoorn M, Van Leeuwen JL, Vanderschoot J, Marani E (2001 b) Electrotonic coupling in a network of compartmental external urethral sphincter motor neurons of Onuf's nucleus. Neurocomputing 38: 647-658.

12. Kinder M, Bastiaanssen EHC, Janknegt RA, Marani E (1995) Neural circuitry of the lower urinary tract; central and peripheral neuronal control of the micturition cycle. Anat Embryol 192: 195-209.

13. Kinder M, Bastiaanssen EHC, Janknegt RA, Marani E (1999) The neuronal control of the lower urinary tract: A model of architecture and control mechanisms. Arch Physiol Biochem 107: 203-222.

14. Holstege G, Griffiths D, de Wall H, Dalm E (1986) Anatomical and physiological observations on supraspinal control of bladder and urethral sphincter muscles in the cat. J Comp Neurol 250: 449-461.

15. Holtstege G, Griffiths D (1990) Neuronal organization of micturition. In: Paxinos $\mathrm{G}$ ed. The human nervous system. Academic Press, Sydney.

16. Kinder MV, Bos R, Willems PJ, Drost MR, Holtzer CA, et al.(1999) Three dimensional registration of mechanical bladder activity using polystyrene fluorescent spheres: A technical note. Arch Physiol Biochem 107: 236-241.

17. Heldoorn M, Van Leeuwen JL, Vanderschoot J (2001a) Modelling the biomechanics and control of sphincters. J Exp Biol 204: 4013-4022.

18. Marani E (2002) The pelvis: another view. Twente University Press, Enschede, The Netherlands.

19. Birder LA, Ruggieri M, Takeda M (2012) How does the urothelium affect bladder function in health and disease. NeuroUrol Urodyn 31: 293-299.

20. Fry CH, Chacko S, De Wachter S, Kanai AJ, Takeda M, et al. (2013) Cell Biology. In: Incontinence. (2013) eds. Abrams P, Cardozo L, Khoury S, Wine A International Consultation on Incontinence, (5th ed).

21. Khater UM, Haddad G, Ghoniem GM (2008) Epidemiology of non-neurogenic urinary dysfunction. In: Pelvic floor dysfunction, Springer-Verlag, London.

22. Kenton K, Lowenstein L, Simmons J, Brubaker L (2007) Aging and overactive bladder may be associated with loss of urethral sensation in women. NeuroUrol Urodyn 26: 981-984

23. Miyazato M, Sasatomi K, Hiragata S, Sugaya K, et al. (2008) GABA receptor activation in the lumbosacral spinal cord decreases detrusor over activity in spinal cord injured rats. J Urol 179: 1178-1183.

24. Fowler CJ, Griffiths D, de Groat WC (2008) The neural control of micturition. Nat Rev Neurosci 9: 453-466.

25. Holtstege G (2005) Micturition and the soul. J Comp Neurol 493: 15-20.

26. Groat de WC (2006) Integrative control of the lower urinary tract: Preclinical perspective. Br J Pharmacol 147: S25-S40.

27. Drake MJ, Apostolidis A, Emmanuel J, Gajewski J, Harrison SCW, et al. (2013) Neurologic urinary and faecal incontinence. In: Incontinence. Eds. Abrams $P$, Cardozo L, Khoury S, Wine A, International Consultation on Incontinence, (5th ed)

28. Schapiro RT (2006) Managing the symptoms of multiple sclerosis. In: Handbook of multiple sclerosis ed. Cook SD, Taylor and Francis, NY.

29. Drake MJ, Apostolidis A, Emmanuel J, Gajewski J, Harrison SCW, et al. (2013) Neurologic urinary and faecal incontinence. In: Incontinence. Eds. Abrams P Cardozo L, Khoury S, Wine A, International Consultation on Incontinence, (5th ed).

30. Andersson KE, Wein AJ (2004) Pharmacology of the lower urinary tract, basis for current and future treatments of urinary incontinence. Pharmacol Rev 56 581-631.

31. Schneider T, Fetscher C, Krege S et al. (2004) Signal transduction underlying carbachol-induced contraction of human urinary bladder. J Pharmacol Exp Ther 309: 1148-1153.

32. Andersson KE (2007) LUTS treatment: Future treatment options. NeuroUrol Urodyn 26: S934-S947.

33. Dong M, Yeh F, Tepp WH et al. (2006) SV2 is the protein receptor for botulinum neurotoxin-A. Science 312: 592-596.

34. Marani E, Pijl MEJ, Kraan MC, Lycklama à Nijeholt GAB, Videleer AC (1993) Interconnections of the upper ventral rami of the human sacral plexus: $A$ reappraisal for dorsal rhizotomy in neurostimulation operations. NeuroUrol Urodyn 12: 585-598.

35. Bastiaanssen EHC, Van Leeuwen JL, Vanderschoot J, Redert PA (1996 a) A myocybernetic model of the lower urinary tract. J Theor Biol 178: 113-133.

36. Bastiaanssen EHC, Vanderschoot J, Van Leeuwen JL (1996 b) State-space analysis of a myocybernetic model of the lower urinary tract. J Theor Biol 180: 215-227.

37. Birder LA, Ruggieri M, Takeda M. van Koeveringe G, Veltkamp S, et al. (2012) How does the urothelium affect bladder function in health and disease. NeuroUrol Urodyn 31: 293-299.

38. Lucchinetti CF, Parisi JE (2006) Pathology: What may it tell us? In: Handbook multiple sclerosis, Taylor and Francis, NY. 